\title{
Modelo de interoperabilidad federado para el intercambio de datos en el sector justicia peruano
}

\section{Federated interoperability model for the exchange of data in the Peruvian justice sector}

\author{
Jaime Salazar-Pimpincos ${ }^{1}$, David Mauricio Sánchez ${ }^{2}$ \\ Universidad Nacional Mayor de San Marcos, Facultad de Ingeniería de Sistemas e Informática. Lima, Perú \\ ${ }^{1}$ Email: jaimesalazar_p@yahoo.es \\ 2Email: dmauricios@unmsm.edu.pe
}

\begin{abstract}
Resumen
La información que fluye en la justicia, particularmente en Perú, no se comparte en tiempo real debido a la heterogeneidad de sus sistemas de información y plataformas, generando excesiva demora del proceso judicial. Por ello, hace más de una década vienen desarrollándose modelos de interoperabilidad para este sector, destacando el modelo europeo e-CODEX que integra a catorce países con sistemas y plataformas diversos y heterogéneos. Sin embargo, estos modelos generan duplicidad de información y presentan dificultad en su implementación por la normatividad legal y estándares exigidos. Aquí, proponemos un modelo de interoperabilidad basado en federación de datos para el sector justicia peruano, este permitirá tener información en tiempo real, distribuida, sin duplicidad, transparente al usuario y de fácil implementación para sistemas y plataformas heterogéneas y heredadas. La propuesta se basa en la arquitectura del e-CODEX, adaptándose y agregando el concepto de federación, consta de tres capas: física, lógica, aplicaciones y un grupo de estandarización. Simulando el proceso de registro de detenidos, donde interactúan cuatro instituciones, demostramos que los tiempos promedios de 20 y 15 días para la operación de registro y el intercambio de información por proceso respectivamente disminuyen a cero, además elimina errores generados por la transcripción de datos.
\end{abstract}

Palabras clave: Sistema de información de Justicia; Interoperabilidad; Sistema Federado; Intercambio de datos en tiempo real.

\begin{abstract}
The information that flows in the justice system, particularly in Peru, is not shared in real time due to the heterogeneity of its information systems and platforms, generating excessive delay in the judicial process. For this reason, they have been developing interoperability models for this sector for more than a decade, highlighting the European model e-CODEX that integrates fourteen countries with diverse and heterogeneous systems and platforms. However, these models generate information duplication and present difficulty in their implementation due to the legal regulations and standards required. Here, we propose an interoperability model based on data federation for the Peruvian justice sector, this will allow to have information in real time, distributed, without duplicity, transparent to the user and easy to implement for heterogeneous and inherited systems and platforms. The proposal is based on the e-CODEX architecture, adapting and adding the concept of federation, it consists of three layers: physical, logic, applications and a standardization group. Simulating the process of registration of detainees, where four institutions interact, we demonstrate that the average times of 20 and 15 days for the registration operation and the exchange of information per process respectively decrease to zero, besides eliminating errors generated by the transcription of data.
\end{abstract}

Keywords: Justice Information System; Interoperability; Federated System; Real-time data exchange.

Dirección: Universidad Nacional Mayor de San Marcos, Facultad de Ingeniería de Sistemas e Informática. Calle Germán Amézaga № 375 , Ciudad Lima 1.

Recibido 01/08/2018 - aceptado 25/08/2018

Citar como:

Salazar J., Mauricio D. Modelo de interoperabilidad federado para el intercambio de datos en el sector justicia peruano. Revista Peruana de Computación y Sistemas 2018 1(2):3-12. http://dx.doi.org/10.15381/rpcs.v1i2.15381

(C) Los autores. Este artículo es publicado por la Revista Peruana de Computación y Sistemas de la Facultad de Ingeniería de Sistemas e Informáticade la Universidad Nacional Mayor de San Marcos. Este es un artículo de acceso abierto, distribuido bajo los términos de la licencia Creative Commons Atribucion - No Comercia_Compartir Igual 4.0 Internacional. (http://creativecommons.org/licenses/by-nc-sa/4.0/) que permite el uso no comercial, distribución y reproducción en cualquier medio, siempre que la obra original sea debidamente citada. 


\section{Introdución}

Desde hace décadas se vienen desarrollando sistemas de información para automatizar diversas labores del sector de Justicia en el mundo, así, se consigue acelerar los procesos, compartir información ahorro de costos y tiempos. Sin embargo, en general, no siguen un plan ni usan la misma tecnología debido a diversos factores como los presupuestos, cambio acelerado de tecnologías, la falta de planes de gobierno de tecnología de información, entre otros [27]. Esto ha ocasionado que la información no se comparta en tiempo real entre sistemas e instituciones del sector de justicia y, como consecuencia de ello, se genera una excesiva demora de los procesos judiciales.

En diciembre del 2012, el INPE informó que, existen 35,892 reos sin sentencia contra 25,498 sentenciados que cumplen una condena, es decir, hay más internos (58\%) en situación de procesado que sentenciados. Así mismo, llama la atención que, en todo el Perú, existan 2,429 reos en cárcel por más de 5 años sin sentencia firme, y además, sólo en Lima existen 22 reos encarcelados por más de 15 años en situación de procesados [2]. Entre los ańos 2007 y 2011 se recibió 112 casos de detenciones arbitrarias por homonimia, debido esto a que, no se toma en cuenta señas y datos particulares que corroboren que dicha persona es la buscada. La única fuente de información para determinar la homonimia es tomada, por los encargados de administrar justicia, del Registro Nacional de Identidad y Estado Civil (RENIEC) [3].

Por ello, desde hace más de una década se vienen desarrollando modelos de interoperabilidad para este sector [7][27], es decir, modelos que permitan a las instituciones del sector de justicia compartir de manera confiable información en tiempo real con la finalidad de acelerar los procesos judiciales. Sin embargo, estos modelos generan duplicidad de información y presentan dificultad en su implementación debido a la normatividad local e institucional y los estándares exigidos para el intercambio de información.

El concepto de federación es una alternativa económica y de fácil implementación para compartir información de forma segura entre sistemas heterogéneos. Por ello, en este trabajo se introduce un modelo, con enfoque federado, de interoperabilidad para intercambiar datos en el sector de justicia peruano, el cual permita tener información en tiempo real, distribuida, sin duplicidad, transparente al usuario y de fácil implementación para sistemas y plataformas heterogéneas y heredadas. El modelo propuesto se basa en la arquitectura del e-CODEX [7], al cual se adapta y agrega el concepto de federación, el mismo que consta de tres capas: física (conectividad), lógica (componentes), y aplicaciones; además de un grupo de estandarización.

Este trabajo está organizado en 6 secciones. En la sección 2, revisamos los conceptos de federación de datos e interoperabilidad, y brevemente el sector justicia peruano. En la sección 3, se presenta una revisión de literatura de marcos de interoperabilidad, así como arquitecturas de interoperabilidad con enfoque federado. El modelo propuesto de interoperabilidad federado para el sector justicia peruano y su validación por simulación es presentados en las secciones 4 y 5 , respectivamente. Finalmente, las conclusiones siguen en la sección 6 .

\section{Marco Teórico}

\subsection{Interoperabilidad}

La interoperabilidad es la capacidad de intercambiar y compartir información entre sistemas, pudiendo utilizar esta información sin requerir una comunicación previa [29]. Además, la Norma Internacional ISO19101:2002 adiciona al concepto el hecho de que se puede controlar el procesamiento cooperativo entre las aplicaciones informáticas [28].

En [24], presentan 15 niveles de interoperabilidad: Semántica, Técnica, Sintáctica, Pragmática, Organizacional, Conceptual, Dinámica, Legal, Social, Esquemática o Estructural, Intracomunitaria, Política-Humana, Internacional, Empírica y Física. En el presente trabajo se ha considerado los Niveles Técnico, Sintáctico, Semántico, Legal y Organizacional.

\subsection{Federación de datos}

La Federación de datos es un conjunto de bases de datos independientes y que cooperan entre ellas. En la federación de datos, los usuarios tienen acceso a los datos, contenidos en cada base de datos independiente, a través de una interfaz virtual como si se tratase de una sola base de datos [25], Los metadatos son la clave de un sistema federado, éstos contienen información acerca de la localización real de los datos, que serán accedidos y usados por el administrador de la federación. En soluciones más avanzadas, los metadatos también pueden contener información detallada acerca de la cantidad de datos que existe en las fuentes de información, así como los caminos de acceso que pueden usarse para acceder a estos. El acceso a los datos, que se mantienen en su base de datos de origen, es en tiempo real. Cuando un sistema requiere información, el administrador de la federación consigue los datos desde las base de datos origen, prepara una vista virtual que será devuelta al sistema solicitante. Las cualidades que tiene esta técnica son la de mantener la información actualizada, gracias a que, el intercambio de datos, se realiza en tiempo real. Es débil, al recuperar y transformar gran cantidad de datos. Se recomienda el uso, de esta técnica, para pequeñas cantidades de información, consultas pequeñas y cuando los datos a integrar sean muy cambiantes, así lo recomienda [26].

\subsection{Sector Justicia del Perú}

El sistema nacional de justicia está conformado por 8 instituciones (Ver figura 1): Tribunal Constitucional [30], Consejo Nacional de la Magistratura [31], 
Congreso de la República [33], Ministerio de Economía y Finanzas [32], Policía Nacional del Perú como parte del Ministerio Interior, Poder Judicial, Ministerio Público - Fiscalía de la Nación y el INPE, administrado por el Ministerio de Justicia. Siendo las cuatro últimas las que intervienen en el proceso judicial, las cuales explicaremos brevemente a continuación.

En el Perú, el Poder Judicial se organiza en niveles. Se inicia con los Juzgados de Paz no Letrados y Letrados que dependen de la Corte Superior que determina el lugar donde van a funcionar, estos juzgados investigan y sancionan casos de faltas menores o que son de rápida solución. Sigue los Juzgados Especializados o Mixtos, que dependen de la Corte Superior y funcionan en una provincia, administran justicia en casos de asuntos civiles, penales, laborales, familia, delitos aduaneros y de delitos tributarios; se establecen en zonas donde no hay Juzgados Especializados y resuelven las apelaciones sobre las sentencias dictadas por los Juzgados de Paz Letrados. La Corte Superior realiza juzgamiento de casos ordinarios donde hay delito, esto es, acciones que son penadas por ley y sancionada con una condena; cabe decir, los delitos se relacionan a daños a la persona o propiedad, tales como fraudes, hurtos, robos, lesiones graves, asesinatos, entre otros. El máximo nivel es la Corte Suprema, donde se atiende las apelaciones dadas en la corte superior [4].

El Estado peruano, tiene como defensor de la legalidad al Ministerio Público, que es un organismo autónomo, titular de la acción penal que hace de oficio o a solicitud del agraviado o la acción popular. Su principal función es, defender los derechos e intereses del ciudadano y la sociedad en general; es el que representa a la sociedad en juicio, defendiendo a la familia, niños, adolescentes e incapaces, manteniendo siempre el interés social, velando por la moral pública, la persecución del delito y la reparación civil [5].

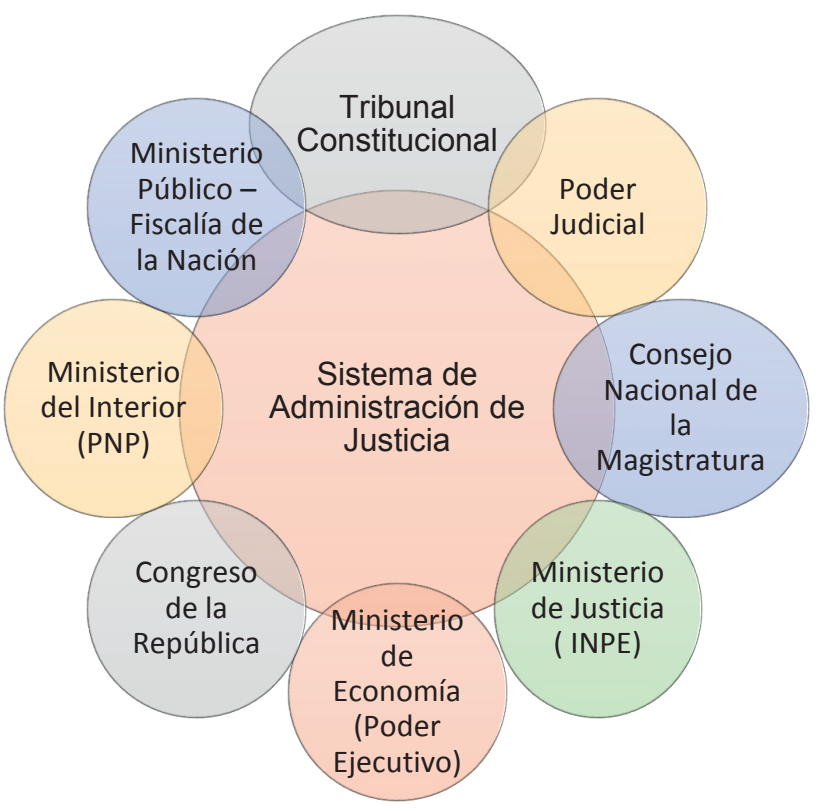

Figura 1. Instituciones del sector justicia peruano
La Policía Nacional del Perú (PNP) es un organismo adscrito al Ministerio del Interior y tiene como funciones las de mantener la seguridad, tranquilidad pública y garantizar el libre ejercicio de los derechos consagrados en la Constitución Política del Perú, así como realizar la prevención de delitos, investigar denuncias, de los ciudadanos o de oficio, sobre delitos cometidos y faltas que se indican en el Código Penal y las leyes especiales; además, debe proteger al niño, adolescente, anciano y mujer en situación de riesgo, además, ejerce también la función de identificación de personas con fines policiales y otras que señala la Constitución y las leyes [17]

El Instituto Nacional Penitenciario (INPE) es un organismo ejecutor, rector del sistema penitenciario nacional peruano que tiene la función de dirigir y administrar el Sistema Nacional Penitenciario, cuyo objetivo es reinsertar social y positivamente a los reos, aseguramiento interno y externo de los centros penales, mejorando condiciones de vida de la población penitenciaria. Otra de las funciones que se tiene es, realizar estudios sobre criminalidad elaborando planteamientos sobre prevención del delito, dar asistencia post penitenciaria, elaborar normas técnicas y administrativas en la planificación y ejecución de proyectos de infraestructura penitenciaria [18].

\section{Revisión de literatura}

\subsection{Marcos de Interoperabilidad en el sector Justicia}

Un marco de interoperabilidad general para gobierno electrónico basado en tecnología de agentes y servicios web, dotado de una arquitectura segura, la cual permitiría atender requerimientos, publicar y prestar servicios es propuesto en [6], pero que aún no ha sido implementada.

En [11], se propone para el gobierno de Indonesia un marco de arquitectura empresarial EAF (Enterprise Architecture Framework) para la interoperabilidad e integración de datos en las aplicaciones de gobierno electrónico, el cual soporte las arquitecturas anteriores existentes; está basado en el protocolo y Lenguaje de marcado extensible (XML) y se distribuye por una red. Por otro lado, en la comunidad europea, desde 2004 se viene implementado un marco de interoperabilidad denominado Comunication Online Data Exchange (e-CODEX), que consta de tres componentes principales (conector, puerta de enlace y la plataforma de entrega) y se basa en bloques de construcción que contiene un e-Id, e-document, e-signature, entre otros; en la actualidad 14 países europeo usan este marco [19].Así mismo, en Brasil, existe el modelo de interoperabilidad e-justice [36], que, según [14], debería mejorar su calidad siguiendo el modelo brasilero de interoperabilidad e-PING [35], y otros como e-PMG [34] y el Marco de Interoperabilidad Europeo (EIF) [29]. 
Del mismo modo, en [12], se analizan los principios de diseño de sistemas y su gestión, tales como el arranque y accesibilidad, adaptabilidad y modularización, ley y tecnología, y base instalada, para comprender su impacto en la capacidad de un sistema y mejorar el acceso a la justicia. En el análisis realizado a seis ejemplos entre europeos y canadienses, se ilustra y desarrolla las formas en que los principios de diseño y su gestión en los sistemas de información (IS) y e-justicia pueden influir en el rendimiento y afectar el valor de la igualdad de acceso a la justicia.

En [7], se analizan los cambios que se están produciendo en el ámbito de Justicia considerando los siguientes principios: complejidad, accesibilidad y alcance de los sistemas; los autores concluyen que debe haber un equilibrio de estos tres principios para el desarrollo de sistemas en el sector de justicia.

\subsection{Arquitecturas con enfoque federado}

Dada la necesidad de integrar los conceptos de administración de bases de datos centralizado y distribuido para aprovechar el control centralizado y el acceso a fuentes de datos heterogéneas distribuidas, en [8], se propone un marco y una arquitectura federada que se construye sobre cuatro componentes principales (Datos, Exportación, Federado, Externo) y seis capas (DB Distribuidas externas, BD Distribuidas internas, BD Federadas, Control, Aplicaciones e interfaces). La propuesta encapsula las componentes dadas en [20] en un esquema de exportación. La propuesta es conceptual y carece de detalles.

Por otro lado, en [9], se propone un marco conceptual federado que considera interfaces locales para codificar y decodificar información usando el estándar High Level Architecture (HLA) a la cual se adiciona la ingeniería inversa del modelo y el enfoque HLA Evolved, que propone mecanismos de interoperabilidad a través de mensajes y sincronización entre sistemas distribuidos.

En [10], se muestra un ejemplo concreto de una arquitectura federada de sistemas de información en el contexto del proyecto Open InFSE[21][37]en el sector salud, para integrar los Sistemas de Información de Salud (HIS, siglas en inglés de Healt Information System) regionales en Italia. Este ejemplo se ha implementado usando la Arquitectura Orientada a Servicios (SOA) por medio de web services (WSs), cuya novedad radica en la propuesta de una infraestructura de notificación de datos clínicos en tiempo real entre HIS federados. Los autores miden el tiempo necesario para la recuperación y notificación de documentos en los escenarios interregional y intra-regional, obteniendo una desviación estándar moderada y considerable (la mitad del tiempo) respectivamente.

De la revisión de la literatura hemos podido advertir que se están realizando muchos esfuerzos para resolver el problema de interoperabilidad. Hoy en día se está buscando que la interoperabilidad esté presente en todos los procesos empresariales e institucionales, donde se requiere que el intercambio de datos sea en tiempo real, seguro y de forma transparente para los usuarios de los sistemas de información.

\section{Modelo Propuesto}

\subsection{MIFID-SJ}

Se propone un Modelo de Interoperabilidad Federado para el Intercambio de Datos para el Sector Justicia Peruano (MIFID-SJ), el cual tiene como objetivo proporcionar a los ciudadanos, instituciones y profesionales del derecho un acceso más fácil a la información, acelerar los procesos para ser más eficiente la colaboración interinstitucional de despachos fiscales, juzgados y autoridades del sector justicia. El modelo considera tres capas: Física (conectividad), Lógica (componentes) y Servicios o Aplicaciones; y un grupo de estandarización (ver figura 2).

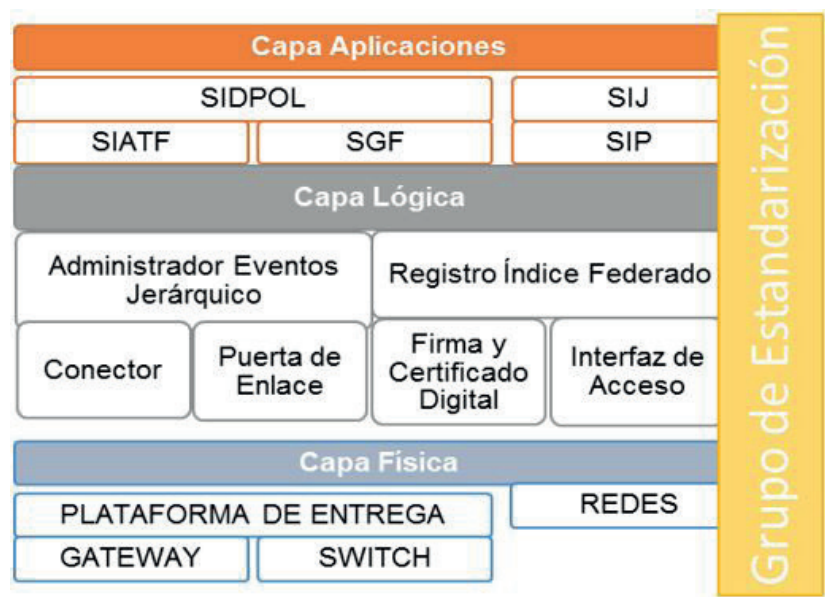

Figura 2. Modelo de Interoperabilidad Federado para el Intercambio de Datos en el Sector Justicia Peruano

La capa física permite la conectividad entre las instituciones del sector justicia. La capa lógica tiene seis componentes (interfaz de acceso, registro de índice federado, conector federado, administrador de eventos jerárquico, la puerta de enlace, firma y certificado digital) y es la que realiza la recepción, transformación y envío de mensajes. La capa de servicios o capa aplicaciones es dada por todas las aplicaciones informáticas locales institucionales al MIFID-SJ que se integrarán. El grupo de estandarización se encargará que definir los estándares y reglas para la interoperabilidad.

El MIFID-SJ se basa en el proyecto a gran escala para el intercambio de datos vía online e-CODEX [22], y a esto se suma el componente federado que ha sido adaptado de la Arquitectura de Interoperabilidad Federada para los Sistemas de Información de Salud en el Proyecto Open INFSE [10].

La implementación de MIFID-SJ permitirá a un usuario enviar datos y documentos (atestado o informe policial, disposiciones fiscales, resoluciones judiciales) 
en tiempo real y de manera segura. Además, las actualizaciones y los registros en los procesos (carpeta fiscal, expediente judicial), así como cada acto o trámite que se registre en los aplicativos informáticos de cada institución del sector justicia, estarán disponibles en tiempo real por todas las instituciones del sector de justicia vinculado a dicho proceso.

\subsection{Capa Física}

La capa física permitirá la conectividad de las instituciones del sector justicia. Este consta de los componentes: Plataforma de entrega, Gateways, Switch, Redes, Servidores (Ver figura 3).

La Plataforma de entrega, es un componente tomado del modelo estándar e-CODEX, que es responsable del transporte seguro y confiable de datos y archivos desde una puerta de enlace a otra. La arquitectura implementada será descentralizada y de surgir una necesidad técnica en el futuro, entonces será considerado como un eje central. Para permitir el acceso a todos los usuarios potenciales, el sistema utilizará Internet con encriptación, usando el protocolo de comunicación https, para garantizar una conexión segura.

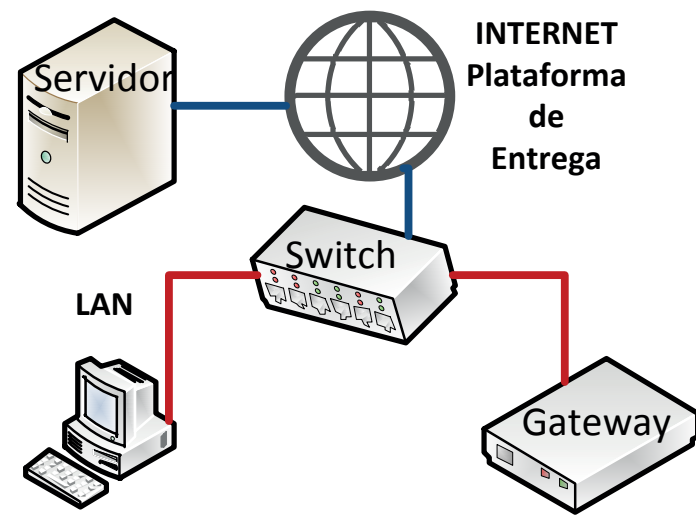

Figura 3. Componentes de la capa física (conectividad)

\subsection{Capa Lógica}

Esta capa tiene por finalidad compartir la información registrada entre las instituciones a través de seis componentes: Conector federado, Puerta de enlace, Firma y Certificado digital, Interfaz de acceso, Registro de índice federado, Administrador de eventos jerárquicos (ver figura 4). Las dos primeras componentes han sido tomadas del modelo estándar e-CODEX y las tres últimas de[10], este último se justifica, porque sus componentes están basados en web services y permiten la implementación del concepto de federación.

Interfaz de acceso es el punto de acceso a las funcionalidades proporcionadas por la capa lógica del modelo, recibe solicitudes de usuarios autorizados y componentes similares de otros sistemas institucionales y las envía a los componentes de los sistemas institucionales miembros del modelo.

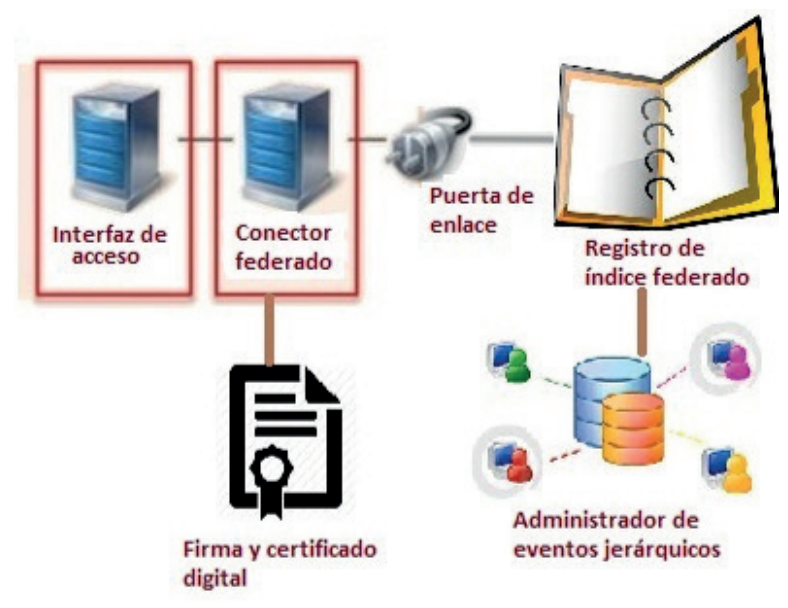

Figura 4. Componentes de la capa lógica (componentes)

Conector Federado realiza tres actividades principales, en primer lugar, transforma los documentos salientes de las aplicaciones informáticas institucionales al estándar MIFID-SJ, así, le agrega una firma y certificado digital electrónico y encripta el documento a enviar. En segundo lugar, transforma los documentos recibidos por la puerta de enlace MIFID-SJ del estándar MIFID-SJ al estándar institucional, verificando la firma y certificado digital electrónico. En tercer lugar, recibe y genera las consultas federadas usando protocolos y traducciones sintácticas y semánticas, y notifica los resultados a cada usuario directamente relacionado con el proceso judicial. Las Instituciones miembros son libres de decidir en qué etapa de su infraestructura llevarán a cabo estas acciones de ser necesarias.

Firma y Certificado digital se trata de un conjunto de datos electrónicos que acompañan o que están asociados a un documento electrónico y que identifican al firmante de manera inequívoca, asegurando la integridad del documento firmado y que es exactamente el mismo que el original, sin haber sufrido alteración o manipulación, siendo los datos únicos y exclusivos, y ello garantiza que el receptor sea el único que pueda entender el mensaje [45]. En el Perú, esta certificación se puede hacer a través de la RENIEC.

Registro de indice Federado permite la consulta de datos del proceso judicial administrados por cada Aplicación Informática al federar los registros institucionales, cada uno de los cuales puede localizar los datos archivados en los repositorios dentro de la institución. Para ello, hacemos uso de cuatro web service, los mismos que interactúan con el registro institucional y reciben solicitudes de la puerta de enlace asociada a la institución:

- Administración de Registro de Índice Federado: Ofrece funcionalidades para definir y administrar federaciones de registros.

- Administración de Consultas: Realiza consultas en su registro asignado o dentro de la federación general de registros. 
- Administración de Metadatos: Gestiona los metadatos relacionados con los documentos del proceso judicial almacenados en el repositorio.

- Administración de eventos: Se utiliza para notificar eventos entre los registros de la federación.

Administrador de eventos jerárquico notifica datos del proceso judicial a todos los usuarios interesados a través de una federación de intermediarios, mediante la adopción de una clasificación jerárquica de eventos basada en el paradigma de publicación/suscripción. Dicha solución de notificación se basa en un servicio de publicación/ suscripción que adopta una topología intermediaria. Este componente se implementa por medio de cuatro web service:

- Administración de registro de publicaciones: Ofrece capacidades de publicación tales como la definición de temas o la publicación de eventos.

- Administrador de intermediarios: Les permite a los usuarios recuperar eventos publicados

- Administrador de suscripciones: Ofrece la posibilidad de registrar predicados de suscripción en el sistema.

- Administración de Federación de Intermediarios: Da los medios para administrar la federación de intermediarios de publicación/suscripción, que enrutan los eventos publicados a los destinos interesados.

Puerta de enlace son canales de comunicación institucionales o sistemas de transmisión de datos entre dos participantes. La puerta de enlace MIFID-SJ están bajo la responsabilidad de cada institución miembro de MIFID-SJ. Se requiere que las puertas de enlace cumplan requisitos de seguridad específicos dentro de su operación, pero también para la comunicación con otros. Estas puertas de enlace actúan como interfaces entre los sistemas institucionales (o el portal del Estado Peruano) y la plataforma de entrega electrónica. En consecuencia, realizan diferentes funcionalidades, como establecer una conexión con otras puertas de enlace y conectores, formatear el contenido de un mensaje para enviar al estándar eBMS3.0 y extraer el contenido de un mensaje recibido de eBMS3.0. El objetivo principal de este componente es llegar a una solución única para construir una plataforma de transporte electrónico para el sector Justicia. Por lo tanto, una solución de plataforma de entrega debe incluir estándares de la industria como ebXML [38] en el área de B2B y ETSI REM [39]. La puerta de enlace tiene las siguientes funcionalidades:

- Controlador de extensiones: Es un conjunto de identificadores de flujo de llamadas a las funciones necesarias para los mensajes entrantes y salientes que extienden las funciones del controlador de servicio de mensajería ebXML (MSH), según lo especificado por OASIS [40].

- Controlador de servicios de mensajería ebXML (MSH): Entidad que puede generar o procesar mensajes que cumplen con servicios de mensajería ebXML de OASIS versión 3.0: Parte 1, capítulos 5 a 8, que especifica las características principales de eBMS 3.0.

- Paquete de mensajes

- Manejo de errores

- Módulo de seguridad

- Módulo de mensajería confiable

Para la puerta de enlace, se sugiere usar el código abierto Holodeck [41].

\subsection{Capa de servicios o aplicaciones}

Esta capa está dada por todas las aplicaciones informáticas de cada institución del sector justicia que se integrarán durante el proceso judicial. Entre ellas se encuentran las siguientes: Sistema de Denuncias Policiales (SIDPOL), Sistema de Apoyo al Trabajo Fiscal (SIATF) o Sistema de Gestión Fiscal (SGF), Sistema de Información Judicial (SIJ), Sistema de Notificaciones Electrónicas (SINOE) [46] y el Sistema de Información Penitenciaria (SIP) y el Portal del Estado Peruano[47].

Las Aplicaciones informáticas deberán tener las funciones principales siguientes:

- Autenticación: Los usuarios de cada institución deben autenticarse para acceder a la AI y poder interactuar con el MIFID-SJ.

- Formulario electrónico: El usuario debe completar un formulario electrónico de acuerdo con la base legal aplicable. Si está permitido por la base legal aplicable, el usuario debería poder agregar los archivos adjuntos necesarios al mensaje en uno de los formatos aceptados.

- Firma electrónica: Para que la parte receptora confíe en el documento, se garantizará mediante la habilitación del usuario para firmar todo el contenido que el caso de uso requiere que se firme, acorde a la normativa legal vigente.

\subsection{Grupo de estandarización}

El sector deberá tener un grupo conformado por representantes técnicos de cada institución del sector justicia. Dicho grupo tiene por objetivo establecer los estándares de interoperabilidad para las tres capas del modelo. Entre estos estándares se deberá considerar las que se señalan a continuación.

Estándares de conectividad referidos a conexiones de red, por cable o inalámbrica; ancho de banda y el ren- 
dimiento, como medida de transferencia de datos en un período de tiempo determinado; y la latencia, referida al tiempo, que incluye la demora de transferencia de datos, desde un punto a otro.

Para los estándares de capa lógica, se deberá tomar en cuenta el estándar eBMS3.0 para formatear el contenido de un mensaje y debe incluir estándares de la industria como eBXML [38], en el área de B2B y ETSI REM [39], y el controlador de servicio de mensajería ebXML (MSH), según lo especificado por OASIS [40].

En la capa de servicios o aplicaciones, se debe considerar la Arquitectura Orientada a Servicios (SOA, por sus siglas en inglés, Service Oriented Architecture), flexible al automatizar la infraestructura y herramientas requeridas, reduciendo costos de integración. SOA, gestiona grandes volúmenes de datos, ocupándose del diseño y desarrollo de sistemas distribuidos [43]. De igual manera se usarán Web Services, que es un software diseñado para intercambiar mensajes, por medio de una red en forma interoperable, transmitidos en formato abierto XML sobre HTTP conjuntamente con otros estándares web [44].

\section{Validación}

La validación del MIFID-SJ se hará a través de una simulación del proceso de detención policial realizada en las diversas Comisarías de Lima - Callao y, de las Divisiones Policiales Especializadas, en el cual se requiere la interacción las instituciones: Policía Nacional del Perú (PNP), Ministerio Público - Fiscalía de la Nación (MPFN), Poder Judicial (PJ) e Instituto Penitenciario (INPE).

\subsection{Proceso actual del registro de detención}

El proceso de detención es una medida cautelar que lo ejecuta la autoridad competente con una duración determinada. La detención tiene como finalidad poner a disposición al detenido ante un juez, o en su defecto puesto en libertad si el plazo máximo de detención expiró, cabe precisar que el plazo de detención se especifica por la ley [48].

En la figura 5 muestra que el proceso comun estipulado por el Nuevo Código Procesal Penal (NCPP) [49] [23] que consta de tres etapas, siendo la etapa de Investigación Preparatoria contemplada en el Art. 321 al $343^{\circ}$ del NCPP donde se registra la información de la detención ya sea por flagrancia o mandato judicial en el Atestado o Informe Policial, según el Art. 65 del NCPP, así como los datos de la identificación del detenido y toda la información relacionada con la detención. La PNP, en virtud del Art. $330^{\circ}$ y $334^{\circ}$ del NCPP, informa a la Fiscalía a través de una notificación la detención efectuada adjuntando los documentos respectivos de la investigación policial y poniendo al detenido a disposición del Fiscal. A partir de este momento el Fiscal apertura una carpeta Fiscal donde se registra la información preliminar del delito y la detención, esta información es registrada en los archivos y repositorios de información del Ministerio Público.

En la etapa intermedia, contemplada en el Art. $344^{\circ}$ al $355^{\circ}$ del NCPP y, luego de realizada la investigación preliminar, el Fiscal, emite la Disposición de Acusación Fiscal, en virtud del Art. 349 del NCPP que se presenta ante el Juez quien genera un expediente judicial para la denuncia presentada y registra la información en los sistemas de información del Poder Judicial, se analiza la información para determinar los elementos probatorios del delito y, si procede la acusación o el sobreseimiento. Como parte de este subproceso se considera dos salidas posibles y no exclusivas.

a. Al inicio o durante este subproceso se determina que existen elementos probatorios suficientes o peligro de fuga o el delito imputado representa una pena mayor a los 4 años o el imputado podría pertenecer a una organización delictiva, el fiscal puede solicitar la prisión preventiva.

b. Al final de este subproceso el fiscal debe dar su disposición indicando si la acusación Procede o no, esto es producto de la investigación realizada, el Fiscal determina como conclusión final: Si hay suficientes pruebas incriminatorias y se debe formalizar la acusación o no hay suficientes pruebas y se determina el sobreseimiento de la acusación.

En la etapa de juzgamiento, contemplada en el Art. $356^{\circ}$ al $403^{\circ}$ del NCPP, el juez, en la audiencia preliminar, define si procede la acusación fiscal. En esta audiencia, las partes exponen sus alegatos al Juez, él se nutrirá de esta información, la evaluará y tomará una decisión respecto a la acusación planteada, finalizando la audiencia. Es preciso indicar que deben estar presentes, en la audiencia, el fiscal y el abogado defensor del acusado. La etapa de juzgamiento, juicio oral, es la más importante del proceso penal. Su objetivo central es que se dicte sentencia sobre la acusación fiscal y sobre los argumentos, alegatos y pruebas expresados por las partes procesales. Esto es, cuando finalizado el Juicio Oral el Juez emite la Sentencia o Mandato Judicial de Absolución, si los elementos probatorios determinan la inocencia del imputado. $\mathrm{O}$ en su defecto, se solicita liberación, Por medio de la notificación del Mandato Judicial (Sentencia de Absolución) se solicita al INPE la liberación en el caso se cumpla la pena establecida o en el caso se trate de una detención por mandato de prisión preventiva y cuyo plazo se ha vencido, el Poder Judicial notifica la excarcelación del detenido.

Actualmente existe demora en la actualización de la información en los aplicativos informáticos de cada institución, debido a la gran carga procesal que tienen, originando con ello que dicha información no esté disponible para los usuarios del sistema de justicia. Cuando el usuario consulta la información sobre un proceso judicial, no tiene la información correcta debido a que 
ésta, es obtenida de diferentes fuentes de datos físicos (atestado o informe policial, carpeta fiscal, expediente judicial) dispersos en diferentes ambientes geográficos.

\subsection{Proceso federado para el registro de detención usando el MIFID-SJ}

En la figura 6 se presenta un flujo de información usando el modelo MIFID-SJ, donde se observa cómo la PNP registra información en sus aplicativo informático $y$ en tiempo real es transmitida hacia el MPFN.

Se explica gráficamente como el usuario de la PNP, desde su sistema SIDPOL interactúa con el usuario del MPFN a través del sistema SGF, el policía debe acceder a su aplicativo informático autenticándose con su usuario y contraseńa, luego se inicia la transacción correspondiente al registro de la identificación del detenido y los datos de la detención, esto se realiza en la etapa de investigación preparatoria del proceso común establecido por el NCPP, inmediatamente, en tiempo real, se transmite el atestado y el informe policial asignándole un identificador de denuncia y el identificador del usuario (policía) hacia el Conector de la PNP, aquí se chequea y se transforma del estándar PNP hacia el estándar MIFID-SJ asignándole la firma y certificado digital. En seguida, en la puerta de enlace de la PNP se incluye documentos y datos al mensaje MIFID-SJ que se enviará por la plataforma de entrega al Ministerio Público-Fiscalía de la Nación (MPFN). La puerta de enlace del MPFN chequea y recupera datos del mensaje MIFID-SJ transmitiéndose al conector MPFN donde se transforma del formato MIFID-SJ hacia el formato estándar MPFN.

Por otro lado, cuando en la institución MPFN, el usuario Fiscal se autentica en el aplicativo informático SGF, sistema de información que le permite operar los documentos y datos creando la carpeta fiscal de la denuncia y transmitiendo los nuevos documentos generados, el identificador del caso SIDPOL, identificador de la carpeta fiscal del SGF y el identificador del Fiscal se verifica el certificado y firma digital y si todo está ok se pasa al conector federado del MPFN. En el conector del MPFN se chequea y se transforma del estándar SGF al MIFID-SJ, agregando el Certificado y firma digital. En la puerta de enlace del MPFN se incluye documentos y datos en un mensaje para ser enviados por la plataforma de entrega hacia la puerta de enlace de la PNP. Así, se cierra el flujo de información de ida y vuelta entre las instituciones PNP y MPFN.

\subsection{Resultados}

Se ha simulado el proceso de detención, estimándose los tiempos de la operación de registro e intercambio de información y cantidad de errores en los datos compartidos. Para tal fin se tomó la medición de los tiempos de cada actividad realizada en un proceso judicial durante un período de 30 días y para validar la calidad de los datos se tomó en cuenta el número de datos errados que fueron enviados por cada institución a través de medios magnéticos o correo electrónico en formato de hoja de cálculo.

Tabla 1. Tiempo de operación de registro de información

\begin{tabular}{lcc} 
Actividades & TA* (días) & $\begin{array}{c}\text { Plazo legal } \\
\text { (días) }\end{array}$ \\
\hline Registrar Detención - PNP & 1 & 1 \\
Registrar Disposición Fiscal - MPFN & 2 & 15 \\
Actualizar Proceso Judicial - PJ & 15 & 90 \\
Registrar Movimientos Penitenciarios - INPE & 2 & 1 \\
Tiempo Total & 20 & 107 \\
\hline
\end{tabular}

* Trámite administrativo

En la tabla 1 se tiene para el registro de la información en los sistemas informáticos institucionales toman 20 días debido a la excesiva carga laboral que se tiene pendiente, esta cantidad no influye en el plazo legal que manda la ley para los actos y trámites propios del proceso judicial que realizaran los operadores de justicia (Policía, Fiscal y Juez).

Tabla 2. Tiempo de intercambio de información

\begin{tabular}{lcc} 
Actividades & TA* (días) & $\begin{array}{c}\text { Plazo legal } \\
\text { (días) }\end{array}$ \\
\hline Datos de Detención - PNP & 8 & 1 \\
Datos de Disposición Fiscal - MPFN & 15 & 15 \\
Datos de Proceso Judicial - PJ & 20 & 90 \\
Datos de Movimiento Penitenciario - INPE & 17 & 1 \\
Promedio & 15 & 107 \\
\hline
\end{tabular}

*Trámite administrativo

La tabla 2 muestra que en promedio se demora 15 días para enviar la información desde una institución a otra y se pueda disponer de ella para su respectiva actualización en los sistemas informáticos respectivos.

Tabla 3. Número de registros con datos errados por cada entrega

\begin{tabular}{lc} 
Actividades de registros & $\begin{array}{c}\text { Nro de } \\
\text { Registros }\end{array}$ \\
\hline Detenciones informadas por la PNP & 150 \\
Disposiciones fiscales informadas por el MPFN & 100 \\
Diligencias del Proceso Judicial informadas por el PJ & 42 \\
Movimientos de internos informadas por el INPE & 40 \\
Promedio & 83 \\
\hline
\end{tabular}

En la tabla 3 se presenta en promedio 83 registros diarios sin ser validados apropiadamente, generándose pérdida de tiempo en la actualización de la información en cada sistema informático institucional.

De la simulación del proceso de registro de detenidos (ver figura 6) que contempla la interacción interinstitucional se muestra que los tiempos promedios de $20 \mathrm{y}$ 15 días para lo operación de registro en los sistemas informáticos y el intercambio de información por proceso judicial respectivamente se reducen a cero, además elimina los errores generados por la transcripción de datos. 


\section{Conclusiones}

En este trabajo se ha propuesto un Modelo de Interoperabilidad Federado para el Intercambio de Datos en el Sector Justicia Peruano (MIFD-SJ), que consta de 3 capas: física (permite la conectividad), lógica (permite compartir información en tiempo real y segura), aplicaciones (dado por todos los sistemas de información del sector de justicia), y un grupo de estandarización. El modelo permite el intercambio y disponibilidad de información en tiempo real, de manera segura, distribuida, sin redundancia transparente al usuario y de fácil implementación para sistemas y plataformas heterogéneas y heredadas. MIFD-SJ está basada en el modelo estándar europeo para el sector de justicia e-CODEX, al cual se agrega el concepto de federación dado en el modelo Open INFSE.

Una simulación de un proceso judicial que involucra la interacción de cuatro instituciones muestra que los tiempos de registros e intercambio de información y los errores generados por la transcripción de datos se eliminan.

\section{Referencias bibliográficas}

[1] 'Informe Estadístico de diciembre de 2012, Instituto Penitenciario del Perú', http://www.inpe.gob.pe/pdf/diciembre2012-e. pdf, accedido 24 Julio 2015.

[2] 'Modelos Mentales y Modelos Conceptuales en la Enseñanza \& Aprendizaje de las Ciencias', https://www.if.ufrgs. br/ moreira/modelosmentalesymodelosconceptuales.pdf, accedido 20 Julio 2015.

[3] 'Defensoría del Pueblo. Nota de Prensa N. ${ }^{\circ} 276 / O C I I / 2012 /$ DP', http://www.defensoria.gob.pe/modules/Downloads/ prensa/notas/2012/NP-276-12.pdf, accedido 24 Julio 2015.

[4] 'Historia del Poder Judicial Peruano', https://historico.pj.gob. pe/conocenos.asp?tema=historia, accedido 20 Junio 2018.

[5] 'Ministerio Público - Fiscalía de la Nación del Perú', http:// www.mpfn.gob.pe/quienes_somos/, accedido 20 Junio 2018.

[6] Fábio Marques et al,: 'A General Interoperability Architecture for e-Government based on Agents and Web Services', Conference: 6th Iberian Conference on Information Systems and Technologies (CISTI), 2011.

[7] Nadia Carboni \& Marco Velicogna,: 'Electronic Data Exchange Within European Justice: e-CODEX Challenges, Threats and Opportunities', International Journal For Court Administration, 2012.

[8] David X. Zhu et al,: 'Towards the Use of both Financial and Non-financial Data for Decision Making: A Conceptual Framework for Federated Database Systems', International Journal of Business and Social Science, Vol. 3 No. 2,2012.

[9] Zhiying Tuet al,: 'A federated approach to develop enterprise interoperability - Building a high-level architecture federated interoperable framework from legacy information systems', International Journal of Computer Integrated Manufacturing, 2014.

[10] Mario Ciampiet al,:'A federated interoperability architecture for health information systems', International Journal of Internet Protocol Technology 7(4):189-202 2013.
[11] Aris Puji Widodo. et al,:"E-Government Interoperability Framework based on a Real Time Architecture', International Journal of Computer Science Issues, 2013.

[12] Giampiero Lupo \& and Jane Bailey: 'Designing and Implementing e-Justice Systems: Some Lessons Learned from EU and Canadian Examples', Laws, 2014.

[13] Tomas Skripcaket al,: 'Creating a data exchange strategy for radiotherapy research: Towards federated databases and anonymized public datasets', Elsevier, Radiotherapy and Oncology, Volume 113, Issue 3, 2014.

[14] L. Silveira et al,: 'Assessing the Brazilian e-Justice Interoperability Model', IEEE Latin America Transactions, Volume: 13, Issue: 5, 2015.

[15] Wout Hofman: 'Federated platforms for seamless interoperability in the Physical Internet', Conference: 2nd International Physical Internet Conference, At Paris, 2015.

[16] Gregory Zacharewicz et al,: 'Model-based approaches for interoperability of next generation enterprise information systems: state of the art and future challenges', Springer Link, Information Systems and e-Business Management, 2017.

[17] 'Ministerio del Interior - Policía Nacional del Perú', https:// www.gob.pe/730-ministerio-del-interior-que-hacemos, accedido 20 Julio 2018.

[18] 'Ministerio de Justicia - Instituto Nacional Penitenciario del Perú', https://www.minjus.gob.pe/historia/, accedido 20 Julio 2018.

[19] 'Cross-border e-Justice in Europe (e-CODEX)' file:///C:/Users/FN/Downloads/PART-2017-315283V1\%20(1).pdf,accedido 06 Agosto 2018.

[20] Sheth, A., Larson, J.: Federated Database Systems for Managing Distributed, Heterogeneous, and Autonomous Databases. ACM Computing Surveys 22(3), 1990. 183-236.

[21] 'OpenInFSE', http://ehealth.icar.cnr.it, accedido 05 Agosto 2018.

[22] 'e-Codex', https://www.e-codex.eu/, accedido 11 Agosto 2017.

[23] 'AMAG guía de actuación del juez en el NCPP', https://www. slideshare.net/hzl2014/amag-gua-de-actuacin-del-juez-enel-ncpp, accedido 12 Agosto 2018.

[24] M.A. Manso, M. Wachowicz, M. A. Bernabé, A. Sánchez y A.F. Rodriguez.: 'Modelo de Interoperabilidad Basado en Metadatos (MIBM)', Depto. de Ingeniería Topográfica y Cartográfica. ETSI en Topografía, Geodesia y Cartografía, Universidad Politécnica de Madrid, 2008. Págs. 3-10.

[25] Luis Fernando Espino Barrios.: 'Sistemas de Bases de Datos Federadas'. Instituto Tecnológico de Costa Rica, 2009.

[26] Colin White, B.R: 'Data Integration: Using ETL, EAI, and EII Tools to Create an Integrated Enterprise', BI Research Webcast de TDWI , 2005.

[27] Mirna Elya, García barrera, Justicia electrónica: 'En busca de la interoperabilidad'. Universidad Autónoma de México. 2016.

[28] 'International Organization for Standarization. ISO 19101:2002', https://www.iso.org/standard/26002.html, accedido 17 Agosto 2018.

[29] 'Guía de implementación de la facilitación del comercio, Comisión económica de las Naciones Unidades para Europa 
(CEP/ONU)', http://tfig.itcilo.org/SP/contents/interoperability. htm, accedido 20 Agosto 2018.

[30] 'Tribunal Constitucional Peruano', http://www.tc.gob.pe/tc/ public/, accedido 20 Agosto 2018.

[31] 'Consejo Nacional de la Magistratura Peruano', https://www. cnm.gob.pe/, accedido 20 Agosto 2018.

[32] 'Ministerio de Economía y Finanzas', https://www.mef.gob. pe/es/, accedido el 20 Agosto 2018.

[33] 'Congreso de la República Peruana', http://www.congreso. gob.pe/?home, accedido 20 Agosto 2018.

[34] 'e-PMG Padrão de Metadados do Governo Eletrônico', https://www.governodigital.gov.br/documentos-e-arquivos/ PMG\%20Versao\%201_1.pdf, accedido 20 Agosto 2018.

[35] 'e_PING Padrões de Interoperabilidade de Governo Eletrônico', https://www.governodigital.gov.br/documentos-e-arquivos/0001_final.pdf, accedido 18 Agosto 2018.

[36] 'e-Justice - Justicia del Gobierno Federal', http://www.justica. gov.br/, accedido 20 Agosto 2018.

[37] 'Progetto Infrastruttura Tecnologica del Fascicolo Sanitario Elettronico', Lineeguida e specifiche tecniche, Dipartimento per la digitalizzazione del la Pubblica Amministrazione e l'innovazione tecnológica \& Dipartimento delle Tecnologie dell' Informazione e delle Comunicazioni. 2010.

[38] 'ebXML', http://ebxml.xml.org/,accedido 21 Agosto 2018.

[39] 'ETSI - REM', https://www.etsi.org/deliver/etsi_ts/102600_ 102699/10264003/02.01.01_60/ts_10264003v020101p.pdf accedido 21 Agosto 2018.
[40] 'OASIS', https://www.oasis-open.org/committees/tc_home. php?wg_abbrev=security, accedido 21 Agosto 2018.

[41] 'HoloDeck', http://holodeck-b2b.org/, accedido el 21 Agosto 2018.

[42] 'Módulos AXIS', https://www.axis.com/es-mx/products/access-control/io-relay-modules, accedido 21 Agosto 2018.

[43] 'Service Oriented Architecture', http://www.opengroup.org/ standards/soa,accedido el 23 Agosto 2018.

[44] 'Web Services', https://msdn.microsoft.com/es-es/library/ bb972248.aspx accedido 23 Agosto 2018.

[45] 'Certificado y firma digital peruano', http://www.reniec.gob. pe/portal/pdf/certificacion/ANEXO6a.pdf, accedido 25 Agosto 2018 .

[46] 'Sistema de Notificaciones Judiciales del Poder Judicial Peruano', https://casillas.pj.gob.pe/sinoe/login.xhtml, accedido 25 Agosto 2018.

[47] 'Portal del Estado Peruano', http://www.peru.gob.pe/, accedido 25 Agosto 2018.

[48] 'Proceso de detención peruano', https://www.unifr.ch/ddp1/ derechopenal/anuario/an_1993_06.pdf , accedido 26 Agosto 2018.

[49] 'Nuevo Código Procesal Penal Peruano', http://spij.minjus. gob.pe/content/publicaciones_oficiales/img/CODIGOPROCESALPENAL.pdf, accedido 26 Agosto 2018. 